\title{
Risques associés aux barrages
}

\section{Risks associated with storage dams}

\author{
André Goubet \\ Ingénieur en Chef des Ponts et Chaussées \\ Président du Comité Technique permanent des Barrages
}

Fin 1977, la France comptait 353 "grands barrages", c'est-à-dire 353 ouvrages mesurant plus de $15 \mathrm{~m}$ de haut au-dessus de leur fondation la plus basse ${ }^{(1)}$.

Ces ouvrages, ainsi que certains barrages de taille plus réduite, font peser sur la population située à l'aval un risque, certes très faible, mais qu'il serait aussi regrettable de négliger totalement que de surestimer.

Bien qu'il soit toujours délicat, notamment au plan psychologique, d'apprécier les risques de très faible probabilité mais de grande ampleur, il est néanmoins préférable de cerner le plus possible le problème plutôt que de prendre des décisions regrettables sur des bases éventuellement grossièrement inexactes ${ }^{(2)}$.

Tel est l'objectif recherché dans cette note.

La quasi totalité des pays du monde possédant des barrages importants sont regroupés dans la "Commission Internationale des Grands Barrages" qui a rassemblé dans un "Registre Mondial" la quasi intégralité des grands barrages existants dans le monde, Chine exclue ${ }^{(3)}$.

Par ailleurs, la Commission a procédé en 1965 à un recensement aussi exhaustif que possible des incidents ou accidents (et donc des ruptures) ayant affecté les grands barrages, recensement ayant porté sur $96 \%$ des barrages existant dans le monde (Chine exclue) à l'époque.

(1) Sur ce total, 202 étaient utilisés à la production d'électricité, parmi lesquels 128 mesuraient plus de $20 \mathrm{~m}$ de haut audessus du fond de la vallée; 64 de ces 128 ayant une capacité de plus de 15 millions de mètres cubes.

(2) Une difficulté analogue se présente d'ailleurs pour les différents risques qu'une terminologie moderne, à mon avis regrettable, qualifié de "risques majeurs".

(3) Ce pays a adhéré récemment et le registre ne fait mention que des 500 ouvrages pris parmi les principaux.
D'autre part, la littérature technique en langue française et anglaise est suffisamment complète pour que l'on puisse admettre qu'aucune des ruptures survenue depuis 1965 n'ait échappé à l'examen des services d'E.D.F. qui ont bien voulu sur ce point compléter mes informations.

Dans ces conditions, on peut comparer le nombre des grands barrages mis en service jusqu'à fin 1977 (le registre mondial s'arrête pour le moment à cette date) et les ruptures survenues pour en déduire une fréquence empirique ${ }^{(4)}$.

Ceci ne signifie évidemment pas que la rupture d'un barrage de moindre importance serait sans conséquence grave $^{(5)}$.

\section{Qu'est-ce qu'un barrage?}

Dans l'csprit des Français un barrage est généralement un ouvrage en béton -encore que beaucoup connaissent

(4) J'ai toutefois, dans la mesure du possible, tenu compte des ruptures constatées jusque fin 1979, en estimant approximativement le nombre actuel des grands barrages en service.

(5) Dernièrement, aux U.S.A., la disparition, à la suite d'une crue survenue au cours de la nuit, du barrage de Kelly Barnes Lake (Toccoa, Georgie) fit 37 victimes bien qu'il n'ait eu que $9 \mathrm{~m}$ de haut;

- toujours aux U.S.A., le barrage d'Austin, bien que n'ayant que $14 \mathrm{~m}$ de haut et ne retenant que 0,75 million de $\mathrm{m}^{3}$ d'eau, entraina la mort de plus de 100 personnes lorsqu'il se rompit le 11 septembre 1911 ;

- enfin, l'une des plus grandes catastrophes des derniers siècles, et sans doute de tous les temps, en matière de barrages, (2 à 4000 morts selon les sources), fut causée par le barrage de South Fork River (Pennsylvanie) qui, bien que classé dans les "grands barrages" n'avait qu'une hauteur modeste: $21,9 \mathrm{~m}$ au-dessus des fondations. 
Serre Ponçon- qui barre une vallée de montagne et permet de produire de l'électricité.

Cette vision, qui n'est pas inexacte en ce qui concerne notre pays est tout à fait erronée au niveau mondial.

En fait, les barrages - et les premiers existaient voici 5000 ans- étaient, à l'origine destinés aux besoins agricoles (ou à l'alimentation en eau des populations) puis, à une époque beaucoup plus récente, sont apparus les barrages associés aux canaux de navigation (pour alimenter les biefs de partage, par exemple) ou à différentes activités industrielles (barrages britanniques du $\mathrm{XIX}^{\mathrm{e}}$ siècle), ensuite à la production d'électricité et, enfin, aux loisirs. Les barrages écrêteurs de crue, qui se multiplient à notre époque (amélioration de la protection des agglomérations) semblent avoir existé dès les premiers siècles de notre ère.

C'est ainsi qu'au début de ce siècle, la moitié des barrages mondiaux ${ }^{(6)}$ étaient situés au Japon, où le plus ancien ouvrage a près d'un millénaire; les barrages japonais étaient tous construits en terre et étaient indispensables à la culture du riz. La Grande Bretagne pour sa part, en abritait près du quart, presque tous en terre également.

Sur 805 barrages antérieurs à 1900, plus de 700 étaient des barrages en terre ou en enrochements, encore que les Romains puis les Arabes, aient réalisé un certain nombre d'ouvrages en maçonnerie(7).

Encore aujourd'hui, la grande majorité des barrages sont des ouvrages constitués par des remblais (terre ou enrochement).

\section{Recensement des "grands barrages" existant dans le monde}

Le Registre mondial mentionnait, à la fin de 1977 , 15628 ouvrages (Chine exclue). Pour quelques rares

(6) En considérant le monde à l'exclusion de la Chine. (7) On peut citer par exemple, pour mémoire, les barragesvoutes suivants :

\begin{tabular}{|c|c|c|c|}
\hline nom & pays & $\begin{array}{c}\text { date de } \\
\text { coństruction }\end{array}$ & hauteur \\
\hline Baume & France & romaine & $\begin{array}{l}12 \mathrm{~m}(?) \text { ruines décou- } \\
\text { vertes au } 18^{\mathrm{e}} \text { siècle }\end{array}$ \\
\hline Dara & Turquie & env. 550 & ? en ruines \\
\hline Kabar & Iran & env. 1300 & $26 \mathrm{~m}$ existe toujours \\
\hline Kurit & Iran & env. 1300 & $\begin{array}{l}60 \mathrm{~m} \text { surélevé de } 3 \mathrm{~m} \\
\text { en } 1850 \text { - en service }\end{array}$ \\
\hline Abbas & Iran & env. 1300 & $\begin{array}{l}20 \mathrm{~m} \text { partiellement } \\
\text { rompu et séparé au } \\
16^{\mathrm{e}} / 17^{\mathrm{e}}\end{array}$ \\
\hline Almansa & Espagne & $1384-1586$ & $\begin{array}{l}15 / 21 \mathrm{~m} \text { encore en } \\
\text { service }\end{array}$ \\
\hline Tibi & Espagne & 1580 à 1594 & $42 \mathrm{~m}$ encore en service \\
\hline Elche & Espagne & 1632 & $23 m$ encore en service \\
\hline $\begin{array}{l}\text { Ponte } \\
\text { Alto }\end{array}$ & Italie & 1611 à 1887 & $\begin{array}{l}5 \text { à } 39 \mathrm{~m} \text {, barrage suré- } \\
\text { levé } 7 \text { fois. }\end{array}$ \\
\hline
\end{tabular}

On peut également signaler en Espagne le barrage Almonacid sur l'Aguasvivas du $13^{\mathrm{e}}$ siècle (barrage en maçonnerie de $29 \mathrm{~m}$ de haut, surélevé ensuite d'un mètre et aujourd'hui envasé). pays toutefois, dont aucun ne comporte un grand nombre d'ouvrages, le recensement s'arrête à fin 1974 , fin 1971, voire fin 69.

A côté des grands barrages proprement dits (c'est-àdire des ouvrages de plus de $15 \mathrm{~m}$ au-dessus des fondations), le Registre peut mentionner des barrages de moindre hauteur, mais présentant un intérêt particulier (longueur, capacité, etc.). Les divers pays usent très différemment de cette faculté. Et si le tiers des 5618 barrages mentionnés par les USA ont moins de $15 \mathrm{~m}$ (ou une hauteur non précisée, mais sans doute faible), les 2003 ouvrages japonais inscrits au registre ont tous plus de $15 \mathrm{~m}$.

De manière à dégager une base de référence homogène, j'ai décompté dans l'annexe 1 , pour chaque pays, les barrages de hauteur inférieure à $15 \mathrm{~m}$, non précisée et supérieure à $15 \mathrm{~m}$.

Les conclusions de cette annexe sont les suivantes:

\begin{tabular}{|c|c|c|c|c|}
\hline & $<15 m$ & $\begin{array}{l}\text { hauteur } \\
\text { indéter- } \\
\text { minée }\end{array}$ & $>15 m$ & Total \\
\hline $\begin{array}{l}\text { USA } \\
\text { Japon } \\
\text { Europe de l'Ouest } \\
\text { Reste du monde } \\
\text { (Chine exclue) }\end{array}$ & $\begin{array}{r}1187 \\
0 \\
310 \\
651\end{array}$ & $\begin{array}{r}717 \\
0 \\
33 \\
44\end{array}$ & $\begin{array}{l}3714 \\
2003 \\
2566 \\
4403\end{array}$ & $\begin{array}{l}5618 \\
2003 \\
2909 \\
5098\end{array}$ \\
\hline Total & 2048 & 794 & 12686 & 15628 \\
\hline
\end{tabular}

Il est en fait à peu près sûr qu'un certain nombre d'ouvrages n'ont pas été mentionnés. Et si la valeur de 15628 est nettement excessive, celle de 12686 est un peu minorée.

\section{Recensement des ruptures de grands barrages}

La Commission Internationale des Grands Barrages a publié en 1974 un document intitulé "Leçons tirées des accidents de barrages" à la suite d'un recensement quasi exhaustif de toutes les ruptures d'ouvrages connues jusqu'en 1965. Les nombreux tableaux statistiques de ce document présentaient un certain nombre d'erreurs matérielles qui ont été redressées par Niklaus Schnitter, dans un article "Sûreté statistique des barrages" paru dans la revue "Wasser Energie Luft" n ${ }^{\circ}$ - 1976.

Par ailleurs, EDF m'a signalé quelques adjonctions ou suppressions complémentaires.

L'annexe 2 est déduite de l'ouvrage de la Commission, compte tenu notamment des remarques de $M$. Schnitter et d'EDF.

L'annexe 3 recense toutes les ruptures postérieures à 1965 dont j'ai pu avoir connaissance.

N'ont pas été pris en compte quelques accidents, parfois graves, ayant affecté des barrages constitués de stériles miniers qui étaient en fait des dépôts mis en place sans faire intervenir la technologie de construction des barrages en remblais.

(8) Europe des 9, Espagne \& Portugal, Autriche et Suisse, Norvège, Suède ot Finlande. 
Par ailleurs certaines ruptures ont sans doute été omises mais elles sont vraisemblablement en nombre très faible.

\section{Risques de ruptures de barrages}

On peut très grossièrement considérer qu'il $\mathrm{y}$ a chaque année 1,5 rupture ${ }^{(9)}$ de barrages pour un ensemble qui approche actuellement 15000 ouvrages et que le risque de rupture est donc de $10^{-4}$ par année et par barrage.

Une telle appréciation est sans utilité pratique parce que trop globale. Il faut examiner les variations de ce risque en fonction de l'âge de l'ouvrage, de sa nature, voire du pays où il a été construit, et du type de risque.

\section{Variation en fonction de l'âge des ouvrages et de leur date de construction}

Les 129 ruptures répertoriées se répartissent ainsi en fonction de l'année de mise en service du barrage d'une part, du nombre d'années qui sépare la mise en service de la rupture, d'autre part.

\begin{tabular}{|c|c|c|c|c|c|c|c|c|c|}
\hline \multirow{2}{*}{$\begin{array}{l}\text { année de } \\
\text { mise en } \\
\text { service }\end{array}$} & \multicolumn{8}{|c|}{$\begin{array}{c}\text { Nombre d'années entre la mise en service } \\
\text { et la rupture }\end{array}$} & \multirow{2}{*}{$\begin{array}{l}\text { Nbre d } \\
\text { barrage } \\
\text { mis en } \\
\text { service } \\
\text { dans la } \\
\text { période }\end{array}$} \\
\hline & $\begin{array}{l}\text { pendant } \\
\text { la cons- } \\
\text { truction }\end{array}$ & $\begin{array}{c}0 \\
\text { à } \\
5\end{array}$ & $\begin{array}{c}6 \\
\grave{a} \\
10\end{array}$ & $\begin{array}{c}11 \\
\text { à } \\
20\end{array}$ & $\begin{array}{c}21 \\
\text { à } \\
40\end{array}$ & $\begin{array}{c}40 \\
\text { à } \\
60\end{array}$ & $>60$ & $\begin{array}{c}(1) \\
\text { total }\end{array}$ & \\
\hline$<1900$ & 2 & 12 & 4 & 5 & 8 & 3 & 4 & 36 & 861 \\
\hline 1900 à 09 & 0 & 7 & 2 & 1 & 0 & 1 & & 11 & 309 \\
\hline 1910 à 19 & 2 & 9 & 1 & 2 & 1 & 5 & & 18 & 534 \\
\hline 1920 à 29 & 1 & 11 & 0 & 3 & 1 & & & 15 & 86 \\
\hline 1930 à 39 & 2 & 1 & 0 & 0 & 0 & & & 1 & 986 \\
\hline $1940 \div 49$ & 2 & 4 & 0 & 1 & 2 & & & 7 & 903 \\
\hline 1950 à 59 & 0 & 5 & 1 & 3 & & & & 9 & 2354 \\
\hline 1960 à 69 & 4 & 5 & 0 & 2 & & & & 7 & 3562 \\
\hline 1970 à 79 & 2 & 4 & 1 & & & & & 5 & $\begin{array}{l}3400 \\
(2)\end{array}$ \\
\hline Total & 15 & 58 & 9 & 17 & 12 & 9 & 4 & 109 & 13778 \\
\hline
\end{tabular}

(1) Pour les ruptures postérieures à la construction.

(2) 2308 sont mentionnés au registre pour la période 1970-77 mais les omissions sont assez fréquentes.

Il est bien évident qu'un certain nombre de ruptures survenues au $17^{\mathrm{e}}$, au $18^{\mathrm{e}}$ ou même au $19^{\mathrm{e}}$ siècle ont ẹté omises. Par ailleurs il y a une certaine ambiguité dans le choix des ruptures pendant la construction : je n'ai pas par exemple tenu compte d'un certain nombre d'incidents mettant en cause la stabilité de louvrage en l'absence d'eau car ils n'entraînent pas de risques pour l'aval.

Ce tableau permet de constater approximativement les fréquences empiriques de rupture suivantes (fréquence par année-barrage).

(9) 20 recensées au cours des 13 dernières années.
Pour tenir compte des 5 ruptures pour lesquelles on ne dispose que de renseignements incomplets, j'ai majoré forfaitairement les valeurs de $5 \%{ }^{(10)}$.

\begin{tabular}{|c|c|c|c|c|c|c|c|}
\hline \multicolumn{8}{|c|}{ Fréquence exprimée en $10^{-4}$} \\
\hline \multirow{2}{*}{$\begin{array}{l}\text { Année de } \\
\text { mise en } \\
\text { service }\end{array}$} & \multicolumn{7}{|c|}{$\begin{array}{c}\text { Nombre d'années entre la mise en service } \\
\text { et la rupture }\end{array}$} \\
\hline & $\begin{array}{c}\text { Pendant } \\
\text { la construc- } \\
\text { tion (1) }\end{array}$ & $\begin{array}{c}0 \text { à } \\
5\end{array}$ & $\begin{array}{l}6 \text { à } \\
10\end{array}$ & $\begin{array}{c}11 \text { à } \\
20\end{array}$ & $\begin{array}{c}21 \text { à } \\
40\end{array}$ & $\begin{array}{c}41 \text { à } \\
60\end{array}$ & $>60$ \\
\hline$<1900$ & 20 & 29 & 10 & 6 & 5 & 2 & 1 \\
\hline 1900 à 09 & 0 & 47 & 14 & 3 & 0 & 2 & \\
\hline 1910 à 19 & 40 & 35 & 4 & 4 & 1 & 5 & \\
\hline 1920 à 29 & 10 & 27 & 0 & 4 & 0,6 & & $x$ \\
\hline 1930 a 39 & 20 & 2 & 0 & 0 & 0 & & $x$ \\
\hline 1940 à 49 & 20 & 9 & 0 & 1 & 1 & $x$ & $x$ \\
\hline 1950 à 59 & 0 & 4 & 0,9 & 1 & & $x$ & $x$ \\
\hline 1960 à 69 & 10 & 3 & 0 & 0,6 & $x$ & $x$ & $x$ \\
\hline 1970 à 79 & 6 & 2 & 0,6 & $\bar{x}$ & $x$ & $x$ & $x$ \\
\hline Total & 11 & 9 & 2 & 2 & 1 & 2 & \\
\hline
\end{tabular}

(1) Fréquence par barrage construit.

Chacune des cases élémentaires de ce tableau correspond à un échantillon d'observation trop réduit pour que l'on puisse assimiler sans précautions, fréquence et probabilité, mais des regroupements permettent de dégager des résultats intéressants (dont certains seront plus amplement développés plus loin, sous le titre "variation en fonction du type de rupture").

a) Ruptures de barrages pendant la construction: on admet volontairement pendant la construction des risques de submersion, car les conséquences sont bien inférieures à celle de la rupture d'une retenue pleine; les précautions prises semblent toutefois s'être accrues progressivement: 1 barrage sur 300 avant 1900,1 sur 600 entre 1900 et 1959,1 sur 1600 depuis 1950 se sont rompus avant la mise en service.

b) Bien que les ouvrages réalisés soient de plus en plus grande taille, l'amélioration de la technologie permet d'obtenir des fiabilités croissantes: la fréquence des ruptures au cours des 10 premières années de vie d'un barrage est passé de $1,6 \%$ (avant 1900) à $1 \%$ (entre 1900 et 1950 ) et $0,2 \%$ (depuis 1950).

c) Approximativement les risques de rupture sont de même importance pendant les 5 premières années que pendant tout le reste de la vie de l'ouvrage. L'on constate que 41 ruptures de barrage en service (sur 110) se sont produites lors du premier remplissage de l'ouvrage ${ }^{(11)}$. Les erreurs commises lors de la conception ou de la construction sont en fait très rapidement

(10) Pour passer du $1^{\text {er }}$ nombre de la colonne 3 du tableau précédent à son homologue du tableau ci-après, on effectue le calcul suivant

$$
\frac{12 \text { ruptures } \times 1,05 \times 10^{4}}{861 \text { barrages } \times 5 \text { ans }}
$$

(11) Le remplissage complet peut n'intervenir que plusieurs années après la mise en service de l'ouvrage. Sur les 41 ruptures survenues au $1^{\text {er }}$ remplissage, 4 sont intervenues $5,7,11$ et 12 ans après la mise en service (Malpasset et Bouzey en France, Puentès en Espagne, Bilberry en Grande Bretagne). 
sanctionnées, soit lors de ce premier remplissage, soit dans les années qui suivent immédiatement: plus du $1 / 4$ des ruptures consécutives à des submersions sont survenues les 5 premières années, ainsi qu'un cinquième des autres ruptures.

d) Il résulte du rapprochement des deux points précédents que les anciens barrages bien que conçus à une époque où la technologie était parfois sommaire, sont sûrs: un ouvrage construit en 1900 et qui a tenu depuis 80 ans est à peu près aussi sûr qu'un barrage mis en service aujourd'hui. Il ne faudrait toutefois pas déduire de cette remarque qu'un barrage ne vieillit pas; nous verrons en particulier que plusieurs des barrages américains rompus en exploitation utilisaient des matériaux périssables (bois, fer...) dont on ne pouvait attendre un bon vieillissement. Par ailleurs, le fait qu'un barrage n'ait pas été submergé pendant ses 80 premières années ne suffit pas à prouver que ses évacuateurs de crue soient suffisants.

\section{Variation en fonction du pays d'implantation}

Parmi les pays possédant de nombreux barrages, l'on peut distinguer 4 grands groupes :

- les U.S.A.

- le Japon,

- l'Europe de l'Ouest (définition donnée ci-dessus), - le reste du Monde (Chine exclue).

La répartition des ruptures survenues sur des ouvrages construits postérieurement à 1900 est la suivante ( $\mathrm{y}$ compris ruptures en cours de construction).

\begin{tabular}{|l|c|c|}
\hline & $\begin{array}{c}\text { Barrages mis en } \\
\text { service entre } \\
1900 \text { et 1974 }\end{array}$ & $\begin{array}{c}\text { Ruptures } \\
\text { correspondantes }\end{array}$ \\
\hline U.S.A. & 3659 & 46 à 48 \\
Japon & 1529 & 4 \\
Europe & 2335 & 7 \\
Reste du Monde & 4303 & 28 à 31 \\
(Chine exclue) & & \\
\hline
\end{tabular}

On ne peut qu'être surpris par la disproportion qui existe entre les fréquences d'accident aux U.S.A. et dans le reste du monde. On peut certes admettre que les ruptures y sont particulièrement bien recensées. Il faudrait toutefois admettre que les $4 / 5$ des ruptures survenues en Europe occidentale depuis le début du siècle aient disparu des souvenirs des spécialistes pour que la situation y soit analogue à celle des U.S.A.

Une telle hypothèse est invraisemblable et il faut bien admettre une différence profonde entre les U.S.A. et l'Europe, le Japon semblant être dans une situation très proche de cette dernière.

Pourquoi une telle disproportion qui ne peut manquer de surprendre, compte tenu de la qualité de la technologie américaine dans de nombreux domaines?

Plusieurs motifs justifient la différence constatée: certaines zones des U.S.A. sont (ou étaient) très peu peuplées et la rupture de certains ouvrages ne présentait pas de conséquences graves; une construction soignée ne s'imposait donc pas; on peut noter à ce sujet que 6 des barrages récents (postérieurs à 1900) se sont rompus
2 fois; il est vraisemblable qu'un tel risque n'a été accepté que dans la mesure où la rupture de ces ouvrages ne portait qu'une atteinte limitée aux biens ou aux personnes situées à l'aval.

On peut à ce sujet noter que le Japon, pays à forte densité de population, a un taux de rupture très voisin du taux européen et beaucoup plus faible que le taux américain.

Par ailleurs, et ceci est particulièrement vrai pour les ouvrages en terre nombreux aux U.S.A. et très sensibles aux déversements par dessus la crête, de nombreux ouvrages sont emportés par les crues; il importe donc d'avoir une bonne connaissance de celles-ci au moment de la conception de l'ouvrage ; or, elles sont particulièrement difficiles à déterminer dans les zones semi arides où la pluviométrie est très irrégulière. C'est précisément ce genre de climat qui règne sur une partie des U.S.A. ouverte depuis peu de temps à la civilisation et aux mesures hydrologiques.

Enfin, mais ceci peut sans doute ètre rapproché des motifs mentionnés ci-dessus, il semble à la lecture de nombreux documents américains que beaucoup d'ouvrages ont été construits dans ce pays avec l'audace propre à l'esprit pionnier, sans la participation du moindre ingénieur.

Le Registre mondial mentionne 55 grands barrages américains antérieurs à 1900, 19 ruptures ont affecté des grands barrages américains de cette période. Même en admettant que la liste des 55 noms soit incomplète, le rapprochement de ces 2 nombres se passe de commentaires!!

A l'opposé, les barrages japonais, dont une grande partie des barrages en terre a été réalisée à une époque où l'on ignorait les théories modernes de la mécanique des sols, ont été bâtís dans un pays de très ancienne civilisation où les crues étaient sans doute beaucoup mieux corinues qu'aux U.S.A. et selon des pratiques rodées par un millénaire d'empirisme; nos constructeurs de cathédrales ont su également réaliser, sans l'appui des techniques de calcul moderne, des ouvrages qui défient les siècles.

Il convient aussi de noter que les barrages construits aux Indes par l'Angleterre ont été affectés de très nombreuses ruptures dont le taux est encore supérieur à celui des U.S.A.

Par ailleurs 7 des 38 ruptures ayant affecté des barrages antérieurs à 1900 sont survenues sur des ouvrages construits par les Français en Algérie, pays dont l'hydrologie était très mal connue à l'époque de la construction des ouvrages.

Si on se limite aux barrages récents, mis en service après 1950, on peut comparer les résultats suivants :

Barrages mis en service de 1950 à 1977 inclus :

U.S.A. : 2516 barrages $^{(12)}$

Japon : 850

Europe : 1549

(12) Il semble en fait qu'une centaine d'ouvrages ne soient pas mentionnés au registre en ce qui concerne les U.S.A. 
dont :

$\begin{array}{lrll}\text { Autriche } & : 36 & \text { Allemagne }: 68 \\ \text { Finlande } & : 35 & \text { France } & : 208 \\ \text { Grande-Bretagne } & : 141 & \text { Italie } & : 204 \\ \text { Espagne } & : 431 & \text { Norvège } & : 166 \\ \text { Portugal } & : 40 & \text { Suède } & : 104 \\ \text { Suisse } & : 91 & \text { Divers } & : 25\end{array}$

Ruptures survenues sur ces barrages:

U.S.A. : 7 ruptures

Japon : 0 "

Europe : 3 "

Pour cette période, dont les états statistiques, limités aux pays considérés, sont sans doute très valables, la différence des résultats constatés est, en dépit de l'importance relativement limitée des échantillons considérés (environ 40000 années pour les barrages aux U.S.A. d'une part, en Europe et au Japon d'autre part), significative.

\section{Variation en fonction des types de barrages}

Les ouvrages en remblais d'une part (généralement barrages en terre, quelquefois barrages en enrochements) et les barrages en maçonnerie de différentes natures, peuvent se rompre selon des mécanismes différents. Les premiers sont en particulier beaucoup plus sensibles que les seconds aux déversements lors des crues et aux écoulements (renards) à travers le barrage.

A première vue, et si l'on considère globalement l'ensemble des barrages mondiaux, les barrages en remblais semblent beaucoup moins sûrs que les autres.

Cette apparence est trompeuse et provient essentiellement du fait que la majeure partie des barrages des Etats-Unis sont en terre, or les ouvrages de ce pays ont été affectés par un nombre de ruptures particulièrement grand.

En fait, si l'on compare les ruptures ayant affecté chacune de ces catégories d'ouvrage aux U.S.A. d'une part, dans l'ensemble "Europe + Japon" d'autre part, on n'observe pas de différence significative ainsi qu'il résulte du tableau suivant :

\begin{tabular}{|l|c|c|c|c|c|c|}
\hline & \multicolumn{2}{|c|}{$\begin{array}{c}\text { Barrages mis en service } \\
\text { entre } 1900 \text { et 1974 }\end{array}$} & \multicolumn{3}{c|}{$\begin{array}{c}\text { Ruptures correspon- } \\
\text { dantes }\end{array}$} \\
\cline { 2 - 7 } & $\begin{array}{c}\text { Rem- } \\
\text { blai }\end{array}$ & $\begin{array}{c}\text { Maçon- } \\
\text { nerie }\end{array}$ & divers & $\begin{array}{c}\text { Rem- } \\
\text { blai }\end{array}$ & $\begin{array}{c}\text { Maçon- } \\
\text { nerie }\end{array}$ & $\begin{array}{c}\text { divers } \\
\text { ou non } \\
\text { précisé }\end{array}$ \\
\hline U.S.A. & 2537 & 608 & 262 & 38 ou & 8 ou 9 & 3 \\
Japon & 851 & 613 & - & 3 & 1 & - \\
Europe & 715 & 1462 & - & 1 & 5 & - \\
\cline { 2 - 7 } & 4103 & $2683^{(1)}$ & 262 & 42 ou & 14 ou & 3 \\
& & & 43 & 15 & \\
\hline
\end{tabular}

(1) En 1830 , sur 500 barrages répertoriés, 16 seulement étaient en maçonnerie; 20 ans plus tard, on n'en comptait encore que 22 sur 579 ; bien que les Romains et les Arabes aient réalisé des ouvrages en maçonnerie depuis plus de 1000 ans, les barrages en maçonnerie réalisés entre 1850 et 1900 étaient des prototypes qui ont malheureusement été à l'origine de certains déboires.

(2) Sans compter Teton, postérieur à 1974.

\section{Variation en fonction du type de rupture}

Les 114 ruptures constatées (en dehors des ruptures pendant la construction) se divisent en 3 grandes catégories :

- ruptures au premier remplissage (44)

- ruptures par submersion (crues) (43)

- ruptures en service normal (27)

A noter qu'aucune n'est imputable à un séisme. (Un tremblement de terre a toutefois rompu en 1978 un remblai important constitué par des stériles miniers et non repris dans nos statistiques).

\section{Ruptures au premier remplissage}

Ces ruptures interviennent genéralement avant 3 ans, mais dans 4 cas, par suite d'un remplissage très lent, elles sont intervenues au bout de $5-7-11$ et 12 ans !

On peut répartir ces ruptures selon la date de la catastrophe et l'emplacement du barrage.

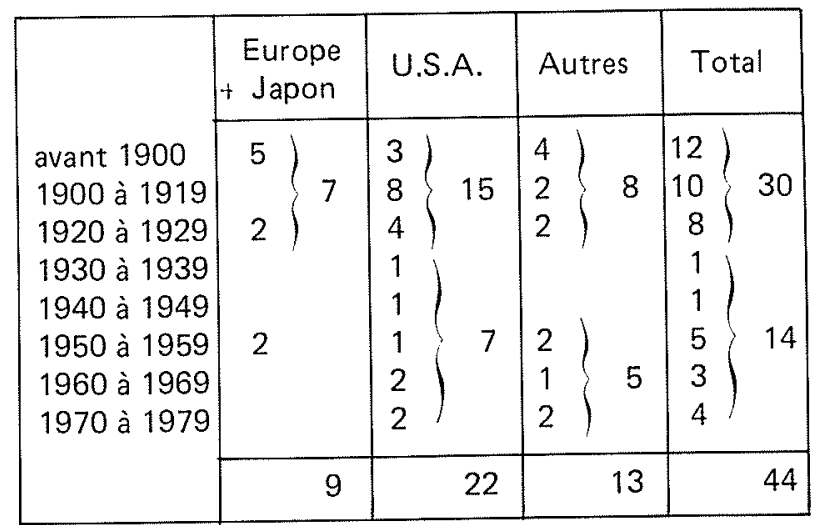

Dans chacune des 3 zones géographiques on constate que les 2/3 des ruptures sont intervenues avant 1929 (et correspondent à environ 3000 barrages réalisés dans le monde) alors que le dernier tiers, survenu après 1930, peut être associé à la construction de 10000 barrages environ. Soit $1 \%$ avant 1930 et $1 \%$ depuis lors.

Le progrès est spectaculaire, qu'il soit dû à une meilleure prévision du comportement des barrages et de leurs appuis ou à une plus grande prudence lors du remplissage.

Mais ici également, et bien que les U.S.A. n'aient pas construit sensiblement plus de barrages que l'Europe de l'Ouest et le Japon réunis (ils en avaient même sensiblement moins en service en 1930) le pourcentage de rupture par barrage mis en service y est près de 3 fois plus important.

On peut également noter que 12 des 14 ruptures survenues depuis 1930 ont affecté des ouvrages en remblais (terre ou enrochement) ; mais depuis 50 ans, la majorité des barrages ont été réalisés en remblais surtout aux Etats-Unis. 


\section{Ruptures par submersion}

Outre l'établissement d'un tableau analogue à celui dressé plus haut, il est intéressant de considérer l'âge des barrages lors de leur rupture :

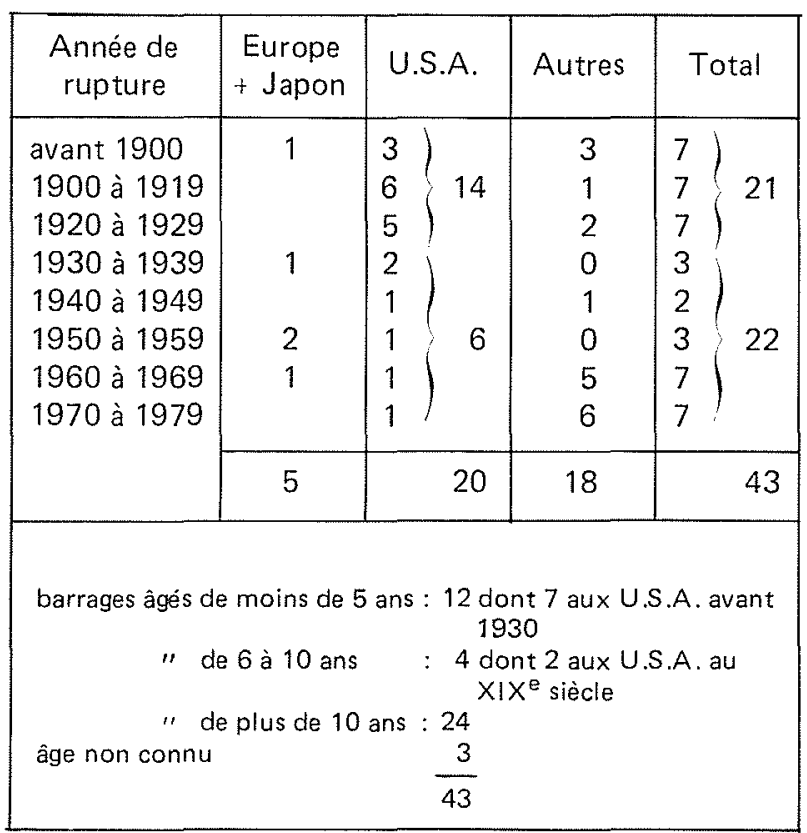

Il convient de rappeler qu'en matière de dimensionnement des évacuateurs, on peut améliorer la situation d'anciens barrages pour tenir compte de l'évolution des connaissances ou des pratiques et qu'il n'y a donc pas lieu de distinguer, parmi les barrages en service aujourd'hui, les ouvrages anciens et les ouvrages récents.

L'on constate que la moitié des ruptures est survenue avant 1930 alors que seul le $1 / 4$ des ouvrages actuels étaient en service; mieux, sur les 21 ruptures constatées avant 1931, la moitié intéresse des ouvrages jeunes (9 de moins de 5 ans, 3 de 5 à 10 ans). Bien qu'une crue tout à fait exceptionnelle puisse survenir immédiatement après la mise en service d'un ouvrage, il est évident que la très grande majorité de ces 12 ruptures correspond à des barrages dont les évacuateurs de crue avaient été notoirement sous dimensionnés, soit par méconnaissance de l'hydrologie, soit par imprudence des projeteurs.

Cette cause de rupture a sensiblement diminué depuis 1930 et il en résulte une amélioration sensible de la sécurité des barrages ${ }^{(13)}$. A noter que l'Europe de l'Ouest et le Japon, pays de vieille civilisation, dont le régime des cours d'eau était mieux connu et où la densité de la population imposait une prudence plus grande, ont toujours été moins sujets aux risques de submersion.

\section{Ruptures en exploitation}

Il convient de noter tout d'abord que 25 des 27 ruptures ont affecté des barrages en remblais (terre ou enrochement) et deux seulement un barrage en béton dont un construit au XIX ${ }^{\mathrm{e}}$ siècle.

(13) Mais le barrage de Bolan, au Pakistan, emporté en 1976 après 15 ans d'exploitation, n'avait pas de déversoir de crue !
Ces ruptures résultent d'une évolution défavorable de certains éléments d'ouvrage (ou de ses appuis) qui avaient subi avec succès un premier remplissage (vieillissement, mauvais entretien, etc.).

Nous allons voir de plus qu'en fait le vieillissement n'apparaît pas comme un motif déterminant et qu'il s'agit souvent d'un défaut d'entretien manifeste d'un type d'ouvrage plus fragile que les barrages en béton.

Ici aussi, il est bon de dresser les 2 tableaux établis comme précédemment.

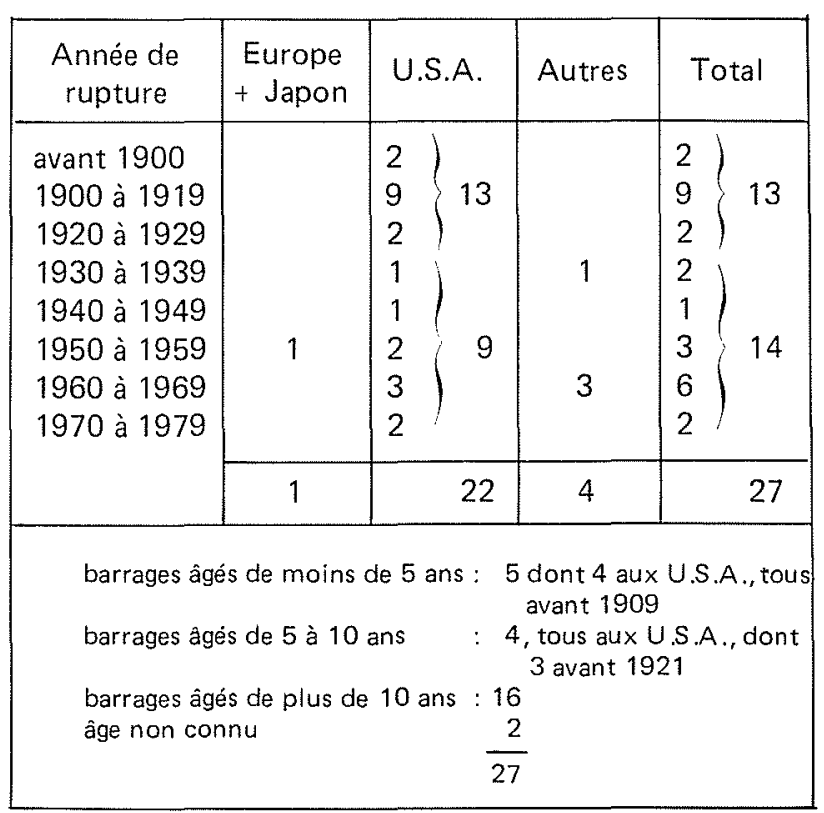

Plus encore que pour les 2 autres catégories d'ouvrages, le caractère spécifique des ouvrages américains est manifeste.

Plus marquant encore est le fait que sur 12 barrages américains rompus avant 1921, 8 avaient moins de 10 ans. En fait ceci correspond à une situation révolue, caractéristique des conditions dans lesquelles s'est ef fectué le développement des U.S.A., et qui n'a plus aujourd'hui qu'un intérêt historique. Des barrages sommaires, dont les responsables n'avaient parfois aucune connaissance technique, ont été mis en place en utilisant des matériaux susceptibles de vieillir rapidement : charpentes en bois ou métalliques, palplanches, gabions en bois... Pour des raisons d'économie ou de rapidité, ces ouvrages étaient en outre parfois dimensionnés au plus juste ${ }^{(14)}$. Ils avaient davantage le caractère de batardeaux provisoires que de barrages définitifs et auraient dû être supprimés rapidement.

C'est pourquoi il est préférable de n'examiner que les 15 ruptures survenues au cours des 50 dernières années. Avec 9 ruptures sur 15, les U.S.A. se mettent ici encore en vedette; ils subissent encore les conséquences (et continueront à les subir) de l'"époque héroïque" car si de nombreux ouvrages se sont rompus avant d'atteindre l'âge de 10 ans, d'autres, conçus et réalisés un peu moins sommairement, mais d'une façon néanmoins peu sûre, continuent à se rompre à un âge,

(14) De même que des barrages en terre à talus très raides et qui se sont également rompus. 
qui pour un barrage, n'a rien de particulièrement élevé. On peut par exemple citer le barrage de Cazdero, à charpente en bois et enrochement, rompu en 1937 à 31 ans (défaillance des appuis des pièces en bois).

Mais le plus souvent la cause de la rupture, que ce soit aux U.S.A. ou ailleurs, se trouve dans un manque d'entretien ou de surveillance; les quelques exemples suivants le mettent en évidence :

- Bon Accord (Afrique du Sud - achevé en 1925 rompu en 1937 - saturation du talus en terre par les fuites provenant d'une conduite de pompage).

- Emery (U.S.A. - achevé en 1850 - rupture rompu en 1966)

- Mill Creeck C (U.S.A. - achevé en 1899 - corrosion rompu en 1957) d'une con-

- Toreson (U.S.A. - achevé en 1898 - duite de rompu en 1953) vidange White Brook (upper) (U.S.A. - achevé en 1942 surélevé en 1949 - rompu en 1972) : le pourrissement d'entretoises en bois entraîne un déplacement des bajoyers de l'évacuateur de crue, et l'apparition d'un renard le long de celui ci.

\section{Conclusions sur les risques de ruptures}

Nous venons de constater que les risques de défaillance s'étaient très sensiblement réduits depuis un siècle. Pour les barrages construits ou en service aujourd hui au Japon et dans l'Europe de l'Ouest, les risques peuvent être très grossièrement appréciés de la façon suivante :

- pendant la construction : le risque est d'environ 0,5 pour 1000 ; cette rupture présente une importance limitée dans la mesure où la cuvette est loin d'être complètement remplie et où l'alerte peut être donnée dans des délais très brefs. Toutefois 2 ruptures survenues récemment en Indonésie et aux Philippines ont causé de nombreuses victimes ;

- pendant le premier remplissage : des progrès spectaculaires ont été faits depuis le début du siècle. Et avec seulement 14 ruptures sur un total de 50 , constatées depuis 50 ans à travers le monde sur les barrages terminés, les ruptures au premier remplissage ont cessé d'être la cause essentielle des rup. tures (avant 1930, il y en avait eu 30 sur 64). Depuis 50 ans, 1 ouvrage sur 1000 s'est rompu au premier remplissage. Le risque est très certainement beaucoup plus faible au Japon ou en Europe de l'Ouest où l'on peut retenir une estimation de 0,5 pour mille;

- par submersion : ici aussi une différence très nette apparaît, à l'échelle mondiale, entre les 50 dernières années et la période précédente, mais surtout à cause du poids des Etats-Unis. L'évolution est moins nette dans les pays de vieille civilisation que constituent le Japon et l'Europe de l'Ouest: 5 submersions ont été observées dans ces pays pour un ensemble qui comportait 805 barrages au début du siècle et 4569 en 1977. Le nombre d'années-barrages observé total dépasse 200000 , ce qui correspond en moyenne à une rupture par submersion tous les 40000 ans. Toutefois, compte tenu des améliorations apportées aux évacuateurs de crue d'un certain nombre de barrages existants et d'une exigence accrue pour les ouvrages récents, le risque actuel est sans doute inférieur;

- par rupture en exploitation : à l'échelle mondiale, l'importance relative de ce type de rupture semble s'accroître par suite de la régression des deux autres: 14 ruptures sur 50 depuis 50 ans contre 13 sur 64 , avant 1930. Mais il convient tout d'abord de remarquer qu'une seule rupture en exploitation sur 27 a affecté un ouvrage en béton ou en maçonnerie (construit d'ailleurs aux U.S.A. au $19^{\mathrm{e}}$ siècle) et que la majorité des barrages français sont en béton. S'il ne faut certes pas en déduire que ce type d'ouvrage est indestructible, et que l'entretien ou la surveillance sont superflus, on peut néanmoins en conclure qu'il est moins fragile que les ouvrages en enrochement et surtout en terre.

Cette rupture peut être associée à un ensemble de 3 à 4000 barrages en béton existants dans le monde et correspondant à environ 100000 années-barrages. On pourrait sans doute prétendre que les barrages européens ou japonais sont mieux conçus et entretenus que le barrage en béton rompu et que leur probabilité de rupture est sensiblement inférieure.

Quant aux 13 ruptures survenues depuis 50 ans sur des ouvrages en remblai, dans la mesure où elles ont affecté pour l'essentiel des ouvrages des U.S.A. souvent réalisés au début du siècle dans des conditions manifestement peu recommandables, il est difficile d'en tirer un enseignement statistique. On peut toutefois observer que dans l'ensemble "Europe de l'Ouest + Japon" on ne connaît que 1 rupture en exploitation pour un ensemble qui comportait 650 barrages en remblai environ au début de ce siècle et près de 2400 en 1974 et qui correspond à environ 150000 années-barrages. Ce qui semblerait montrer que, dès lors qu'ils sont bien entretenus, les barrages en remblai ne présentent eux aussi que des risques insignifiants ;

- risque total : à un risque de rupture pendant la construction de 0,5 pour mille barrages réalisés, on peut donc aujourd'hui, en Europe de l'Ouest et au Japon, ajouter un risque de rupture au premier remplissage de 0,5 à 1 pour mille également, puis en cours de service un risque de rupture par submersion inférieur à 1 pour 50000 annéesbarrages et un risque de rupture pour une autre cause de l'ordre de 1 pour 100 à 200000 annéesbarrages, soit en tout, un risque global de l'ordre de 1 pour 50000 années-barrages.

Ceci correspond, pour la France, à une rupture de grands barrages tous les 1 ou 2 siècles (une fois la première mise en eau effectuée) et, pour l'Europe de l'Ouest, tous les 20 ans ${ }^{(15)}$. Il y a une bonne concordance entre cette observation et le fait que les deux dernières ruptures d'ouvrages survenues après la mise en service aient eu lieu en Europe de l'Ouest en 1944 (Bilberry en Angleterre) et en 1935 (Zerbino en Italie).

Il faut évidemment tenir compte en outre du nombre de ruptures susceptibles d'affecter de nouveaux barrages pendant leur construction et leur mise en eau.

(15) Pour les seuls "grands" barrages. 


\section{Victimes causées par les ruptures}

A ma connaissance, aucun recensement exhaustif n'a été tenté, mais un certain nombre d'éléments mérite d'être signalé : sans aller jusqu'à prétendre que la hauteur et la capacité du barrage sont sans importance sur le nombre de victimes, on peut noter que le barrage de South Fork River à l'origine de la catastrophe la plus grave (2 à 4000 morts) avait seulement $21,9 \mathrm{~m}$ audessus de la fondation la plus basse et ne contenait que 18,5 millions de $\mathrm{m}^{3}$ alors que la rupture du barrage de Teton - analogue à celui de Serre Ponçon- n'a fait que 11 victimes.

Le peuplement de la vallée située à l'aval joue évidemment un rôle essentiel, mais il semble qu'en fait l'élément fondamental de la dimension de la catastrophe soit l'existence ou non d'une alerte préalable, même si elle n'est donnée que très peu de temps avant.

Pendant la guerre 2 barrages ont été détruits dans la Rhur ; la rupture du barrage de la Möhne fit 1200 morts (116 millions de $\mathrm{m}^{3}$ libérés, débit initial estimé à $8000 \mathrm{~m}^{3} / \mathrm{s}$ ), celui de l'Eder, 68 seulement ( 160 millions de $\mathrm{m}^{3}$ libérés, débit initial estimé à $8500 \mathrm{~m}^{3} / \mathrm{s}$ ), mais dans le second cas, il semble que la population ait pu être partiellement alertée, les réseaux téléphoniques étant restés pour partie en service.

On peut de même opposer les 400 à 2000 morts causés par la rupture du barrage de San Francis à minuit (en 1927) et les 11 morts victimes du Teton qui s'est rompu à midi et pour lequel l'alerte a été donnée à temps.

Les accidents les plus catastrophiques dont j'ai trouvé mention sont les suivants :

Macchu 2 (Inde)

- rompu en août 1979 par submersion

- $26 \mathrm{~m}$ au-dessus de la fondation

$-100 \mathrm{hm}^{3}$

- plusieurs milliers de morts.

South Fork River (Pennsylvanie - USA)

- rompu vers $3 \mathrm{~h}$ le $31 / 5 / 1889$

- 21,9 m au-dessus de la fondation la plus basse

- $18,5 \mathrm{hm}^{3}$ de capacité

- barrage en terre emporté par une crue

- 2000 à 4000 morts selon les auteurs .

Iruka (Japon)

- rompu en 1868

-1200 morts.

Saint Francis (Californie - USA)

- rompu à minuit le 12/3/1928

$-47 \mathrm{hm}^{3}$

- barrage-poids en béton

- 400 à 2000 morts selon les auteurs.

Oros (Brésil)

- rompu en 1960

- 1000 morts.

Puentes (Espagne)

- rompu le 30/4/1802

- $50 \mathrm{~m}$ au-dessus du sol

- barrage-poids

- 608 morts.
Malpasset (France)

- rompu le $2 / 12 / 1959$ à $21 \mathrm{~h}$

- 66,5 $\mathrm{m}$ de haut

- voûte

- 421 morts.

Austin (USA)

- rompu le 11/9/1911

$-14 \mathrm{~m}$ de haut

$-0,75 \mathrm{hm}^{3}$

- barrage-poids

- 80 à plus de 700 morts selon les sources .

Vega de Terra (Espagne)

- rompu en 1959

$-34 \mathrm{~m}$ de haut

$-8 \mathrm{hm}^{3}$

- barrage à contreforts

- 144 à près de 400 morts selon les sources.

Quebrada La Chapa (Colombie)

(p.m. : barrage naturel)

- rompu en 1963

- 250 morts .

Hyokiri (Corée)

-. rompu en 1961

-250 morts.

Dale Dyke (G.B.)

- rompu en 1864

- 250 morts .

Canyon Lake (USA - Dakota du Sud)

- rompu en juin 1972 (à la suite de fortes pluies)

- 230 morts.

Sempor (Indonésie)

- rompu en décembre 1967 (en cours de chantier)

- barrage en enrochement

-200 morts.

Gleno (Italie)

- rompu le $1 / 12 / 1923$

- $15 \mathrm{~m}$ au-dessus du sol (22 au-dessus des fondations)

- barrage à contreforts

- plus de 100 à 600 morts selon les sources.

Buffalo Creeck (USA - Virginie occidentale)

- rompu en février 1972 suite à fortes pluies

- (retenue d'eau usée)

- 125 morts .

Bouzey (France)

- rompu le $27 / 4 / 1895$

- $15 \mathrm{~m}$ au-dessus du sol (22 au-dessus des fondations)

- barrage-poids

- 86 à plus de 100 morts selon les sources .

Babü Yar (URSS)

(p.m. : barrage vraisemblablement $<15 \mathrm{~m}$ )

- rompu en 1961

- 145 morts.

Nanaksagar (Inde)

- rompu de 7/9/1967

- 15,6 m de haut

- barrage en terre

- 100 morts.

Del Monte (Colombie)

- rompu en octobre 1976

- 80 morts. 
Santo Thomas (Philippines)

- rompu en 1976 (crue sur le chantier)

- barrage en terre

- 80 morts.

A ces catastrophes, on peut ajouter celle de Vajont (Italie) qui, bien qu'elle ne soit pas imputable à une rupture de barrage, causa environ 2000 morts.

J'emprunte à Niklaus Schnitter le passage suivant :

"La gravité des ruptures de barrage peut se juger au nombre de morts. Le nombre de morts n'était indiqué que dans $40 \%$ des cas ${ }^{(16)}$, mais on peut supposer que les grandes catastrophes n'ont pas été passées sous silence et qu'elles sont toutes comprises dans ces $40 \%$. Il est possible, dans ces conditions, d'établir une relation entre le nombre des grandes catastrophes et le nombre total de ruptures, D'autre part, parmi ces $40 \%$ de cas de rupture, la moitié ne fit aucun mort d'après les compte-rendus. Grâce à ces éléments d'information, il a été possible de déterminer la distribution de fréquence des morts par rupture de barrage" :

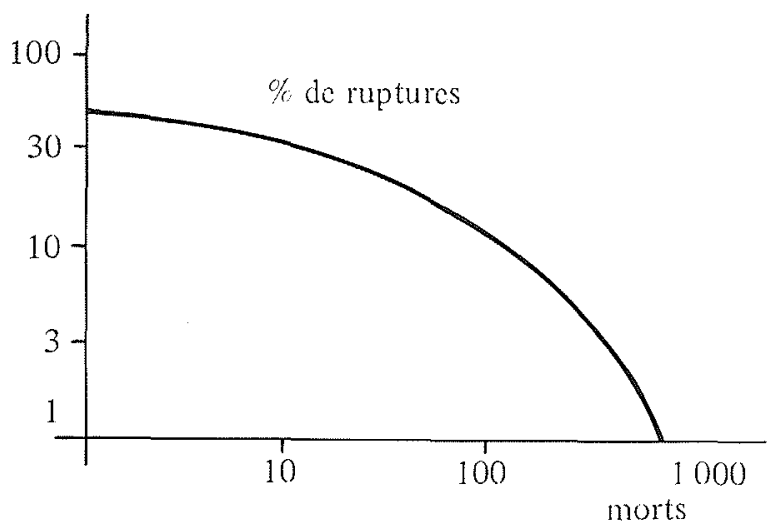

Ce graphique, valable à l'échelle du monde, est peutêtre optimiste en ce qui concerne les barrages construits dans des pays à densité de population élevée.

On doit toutefois noter qu'une rupture de barrage est très loin d'entraîner nécessairement des pertes en vie humaine et que la rupture du barrage de Malpasset, qui a endeuillé notre pays, constitue environ la $7^{\mathrm{e}}$ catastrophe (par ordre d'importance) connue au monde.

Bien que cela n'ait pas grand sens, on pourrait déduire de ce graphique qu'en moyenne une rupture de "grand barrage" est à l'origine d'une cinquantaine de mort ${ }^{(17)}$.

(16) Dans les fiches relatives aux accidents rencensés en 1965 par la Commission Internationale des Grands Barrages.

(17) On peut également rappeler à combien ont été évalués les dom mages matériels associés à quelques ruptures :

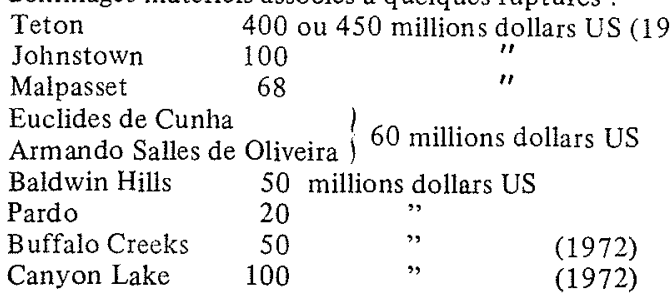

\section{Signes précurseurs des ruptures}

Nous venons de voir le rôle fondamental que peut jouer l'alerte pour réduire les pertes en vies humaines. Il est donc important de savoir si un barrage "prévient" de sa rupture.

Dans de nombreux cas, personne ne se trouvait sur les lieux dans les heures qui ont précédé la rupture (ou, ce qui revient au même, les témoins sont morts dans l'accident) et l'on ne connaît pas, ou mal, l'enchaînement des circonstances qui ont conduit à la catastrophe.

Nous examinerons successivement les 3 grandes catégories de rupture ( 1 ère mise en eau, submersion, rupture en cours d'exploitation) sachant que les ruptures en cours de travaux ne causent généralement que peu de morts (en dépit des 2 exemples malheureux de Sempor et de Santo Thomas).

1) Première mise en eau : à condition de procéder à un remplissage progressif et de suivre attentivement l'évolution du barrage, de ses appuis et des fondations, on devrait vraisemblablement constater, au moins quelques heures à l'avance, l'approche d'une éventuelle rupture. Quelques cas célèbres permettent de le penser :

- pour le barrage du Puentes, en 1802, un messager court avertir la population mais est rattrapé par les flots,

- au barrage de St-Francis (1928) les fuites augmentent fortement pendant l'après-midi (destruction à minuit) et notamment le barrage se vide de $10 \mathrm{~cm}$ dans la $1 / 2$ heure précédant la rupture,

- pour Malpasset (1959) l'on n'avait pas su interpréter, à l'époque, les signes avertisseurs, mais ceux-ci ont manifestement existé (de plus le remplissage a été rapide),

- au barrage de Teton (1976) les ouvriers présents ont constaté le début de la destruction et ont eu le temps de donner l'alerte.

2) Submersion : le signal d'alerte est essentiellement constitué par la pluie ; dans les bassins importants la montée de la crue est suffisamment lente pour que l'alerte soit donnée en temps utile; pour les barrages contrôlant de petits bassins versants (de l'ordre de la centaine de kilomètres carrés) et qui souvent ne sont pas gardiennés, l'alerte sera d'autant plus difficile à organiser que la crue peut être consécutive à un orage relativement localisé au bassin de la retenue et sur lequel l'attention n'est pas immédiatement attirée.

Dans ce cas, et surtout s'il s'agit d'ouvrage en remblai, les ouvrages d'évacuation doivent être dimensionnés d'une façon particulièrement prudente et ne pas comporter d'organes mobiles (à moins que ceux-ci ne soient automatiquement asservis au niveau amont).

Il est toutefois à noter que même les ouvrages en remblai supportent souvent des déversements importants avant de se rompre : à South Fork River (en 1889 aux U.S.A.) le barrage déverse plusieurs heures avant de se rompre en 1/2 heure; à Euclides de Cunha (en 1977 au Brésil) un barrage homogène en terre déverse $7 \mathrm{~h}$ avant de se rompre, la lame déversante atteignant au moins $1,26 \mathrm{~m}$.

3) Rupture en service: on connaît quelques rares cas, parmi les incidents n'ayant pas donné lieu à rupture, où les phénomènes dangereux se sont manifestés prati- 
quement instantanément (glissement du talus aval d'un barrage en terre aux U.S.A., éboulement rocheux à l'aval d'un barrage en Autriche). Toutefois, dans de nombreux cas des signes précurseurs se sont manifestés. Ils se manifesteraient aujourd'hui, compte tenu du développement des moyens de contrôle et de surveillance, dans la très grande généralité des cas. On peut par exemple citer les cas des ruptures suivantes:

- Baldwin Hills (1963 - U.S.A.) rompu à la suite de mouvements de terrains qui se sont étendus sur plusieurs années;

- Greenlick (1904 - U.S.A.) rompu à la suite d'une fuite importante qui avait constamment augmenté pendant ses 3 années de vie;

- Julesburg (1910 -- U.S.A.) idem;

- Sinker Creek (1943 - U.S.A.), fuite importante et saturation progressive du talus aval;

- La Laguna (1969 - Mexique) rompu à la suite d'un lent mouvement du sous-sol;

- Walter Bouldin (1967 - U.S.A.) rompu à la suite d'un glissement progressif du talus amont.

\section{Conclusion}

Cette étude permet d'affirmer qu'en moyenne un barrage est un ouvrage très sûr qui ne fait pas courir de risque déraisonnable à la population, même dans les pays qui attribuent un prix élevé à la vie humaine.

Il y a lieu en particulier de noter les progrès sensibles constatés depuis une cinquantaine d'années.

Mais il ne faudrait pas en tirer une fausse impression de sécurité et oublier que ce résultat n'a été atteint que grâce aux grandes précautions prises à tous les stades : conception, réalisation et exploitation.

Des progrès peuvent certainement encore être obtenus; il convient donc de poursuivre les efforts actuels et de ne pas relâcher l'attention apportée tant à la construction qu'à l'exploitation des barrages.

\section{ANNEXE 1 \\ ANALYSE DU REGISTRE MONDIAL DES BARRAGES}

Le Registre Mondial des Barrages regroupe, à fin 1977 , tous les grands barrages des 71 pays membres de la Commission Internationale des Grands Barrages (c'est-àdire de la quasi totalité des pays possédant de tels ouvrages) et même d'un certain nombre de pays non membres (Angola, Mozambique,...). Toutefois dans le cas de la Chine, qui n'a adhéré qu'en 1974 à la Commis. sion, le Registre ne comporte qu'une liste de 500 ouvrages pris parmi les plus importants.

Ce Registre ne comporte en principe que les ouvrages "de plus de $15 \mathrm{~m}$ de hauteur, mesure prise de la partie la plus basse de la surface générale des fondations jusqu'à la crête".

Toutefois des barrages de 10 à $15 \mathrm{~m}$ peuvent figurer s'ils présentent un intérêt particulier (longueur, largeur, capacité importante, etc...).
L'on constate qu'en fait les différents états ont utilisé de façons très diverses cette possibilité d'extension, certains mentionnant même des ouvrages de moins de $10 \mathrm{~m}$, exclus en principe du Registre. Si la totalité des 2003 barrages japonais ont plus de $15 \mathrm{~m}$, une large part des 5618 ouvrages américains ont moins de $15 \mathrm{~m}$ (ou ont une hauteur non précisée).

Pour procéder à une étude statistique tant soit peu précise, il était donc nécessaire de ne retenir, dans la liste du Registre mondial, que les ouvrages d'une hauteur effectivement égale ou supérieure à $15 \mathrm{~m}$.

Cette annexe présente les résultats obtenus.

\section{Etats Unis}

Les Etats-Unis occupant le $1 / 3$ du Registre mondial (hors Chine) et ayant présenté par ailleurs une très forte proportion de petits ouvrages, il convenait d'examiner plus particulièrement les tableaux correspondants qui permettent de dégager les résultats ci-après :

\begin{tabular}{|c|r|r|r|r|}
\hline $\begin{array}{c}\text { années de mise } \\
\text { en service }\end{array}$ & $<15 \mathrm{~m}$ & $\begin{array}{c}\text { barrages de } \\
\text { hau teur non } \\
\text { précisée }\end{array}$ & $\geqslant 15 \mathrm{~m}$ & Total \\
\hline avant 1900 & 37 & 42 & 55 & 134 \\
1900 à 1909 & 47 & 37 & 106 & 190 \\
1910 à 1919 & 47 & 69 & 212 & 328 \\
1920 à 1929 & 54 & 47 & 302 & 403 \\
1930 à 1939 & 57 & 101 & 285 & 443 \\
1940 à 1949 & 53 & 38 & 238 & 329 \\
1950 à 1959 & 244 & 57 & 576 & 877 \\
1960 à 1969 & 491 & 257 & 1142 & 1890 \\
1970 à 1977 & 157 & 69 & 798 & 1024 \\
Total & 1187 & 717 & 3714 & 5618 \\
\hline
\end{tabular}

Ainsi plus du tiers des barrages des Etats-Unis inscrits au Registre ont moins de $15 \mathrm{~m}$ ou sont de hauteur non précisée (et, semble-t-il, la plupart du temps inférieure à $15 \mathrm{~m})$.

Le redressement à opérer est particulièrement important, s'agissant des barrages antérieurs à $1900^{(18)}$.

Signalons par ailleurs que les U.S.A. ont présenté à la Commission un tableau statistique portant théoriquement sur les barrages du Registre et les regroupant par type, âge et hauteur. En fait cette statistique porte sur un total de 6433 ouvrages (qui comprend manifestement de nombreux barrages inférieurs à $15 \mathrm{~m}$ ) contre 5366 inscrits au Registre. Aucune explication de cette différence n'est fournie.

L'analyse des indications figurant au Registre permet de dresser, pour les seuls ouvrages de plus de $15 \mathrm{~m}$ le tableau récapitulatif suivant, à comparer au tableau fourni par les U.S.A. et reproduit ensuite.

(18) Par ailleurs les tableaux signalent la construction de barrages de

- $274 \mathrm{~m}$ en 1850 (pour une capacité de $800000 \mathrm{~m}^{3}$ )

- $122 \mathrm{~m}$ en 1884 (pour une capacité de $200000 \mathrm{~m}^{3}$ )

- $84 \mathrm{~m}$ en 1887 (pour une capacité de $1,5 \mathrm{millions} \mathrm{de}^{3}$ )

- $205 \mathrm{~m}$ en 1896 (pour une longueur en crête de $75 \mathrm{~m}$ )

Ces valeurs sont évidemment erronées et j'ai supposé qu'il fallat les diviser par 10. D'autres erreurs de cette nature subsistent, tel vraisemblablement ce barrage de $82 \mathrm{~m}$ de haut et de $416 \mathrm{~m}$ de longueur en crête, $\mathrm{mis}$ en service en 1905 et ne contenant que 7 millions de $\mathrm{m}^{3}$. 


\begin{tabular}{|c|c|c|c|c|c|c|c|c|c|c|c|c|c|c|c|c|c|c|c|c|c|}
\hline \multirow{2}{*}{ T y p e } & \multirow{2}{*}{ Tot81 } & \multicolumn{5}{|c|}{ jususqu'_en 1950} & \multicolumn{5}{|c|}{ 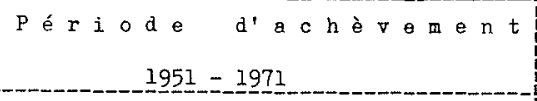 } & \multicolumn{5}{|c|}{$1972=1974$} & \multicolumn{5}{|c|}{ _1975_= 1977} \\
\hline & & & & & & & & & $h a u$ & $t e v r$ & & & & & & & & & & & \\
\hline & & $\leqslant 30$ & $31-60$ & $61-100$ & $101-150$ & $>150$ & $\leqslant 30$ & $31-60$ & $61-100$ & $101-150$ & $>150$ & $\leqslant 30$ & $31-60$ & $61-100$ & $101-150$ & $>150$ & $\leqslant 30$ & $31-60$ & $61-100$ & $|101-250|$ & $>150$ \\
\hline $\begin{array}{ll}\text { terre } & \text { (1) } \\
\text { enrochement }\end{array}$ & $\begin{array}{r}2524 \\
118\end{array}$ & $\begin{array}{r}404 \\
25\end{array}$ & $\begin{array}{r}140 \\
17\end{array}$ & $\begin{array}{r}25 \\
5\end{array}$ & $\begin{array}{l}3 \\
1\end{array}$ & $\overline{-}$ & $\begin{array}{rl}1 & 150 \\
12\end{array}$ & $\begin{array}{r}199 \\
20\end{array}$ & $\begin{array}{l}63 \\
13\end{array}$ & $\begin{array}{l}9 \\
8\end{array}$ & ? & $\begin{array}{r}286 \\
7\end{array}$ & $\begin{array}{r}45 \\
3\end{array}$ & $\begin{array}{r}12 \\
1\end{array}$ & $\begin{array}{l}3 \\
2\end{array}$ & $=$ & 142 & $\begin{array}{r}28 \\
2\end{array}$ & $\begin{array}{l}6 \\
1\end{array}$ & 1 & 1 \\
\hline terre/enroch. (3) & 153 & 25 & 10 & 4 & $i$ & - & 35 & 24 & 8 & 1 & - & 18 & 8 & 4 & $i$ & - & 4 & $\begin{array}{l}5 \\
7\end{array}$ & $\begin{array}{l}1 \\
2\end{array}$ & $\begin{array}{l}1 \\
1\end{array}$ & $=$ \\
\hline remblais $(1+2+3)$ & 2795 & 454 & 167 & 34 & 5 & - & 1197 & 243 & 84 & $\overline{18}$ & 7 & 311 & 56 & $\overline{17}$ & $\overline{6}$ & - & 146 & 37 & $\overline{9}$ & $\overline{3}$ & $\overline{1}$ \\
\hline poids (4) & 376 & 149 & 86 & 25 & 3 & 2 & 59 & 21 & 17 & 4 & - & 2 & 3 & - & 1 & 1 & - & 2 & 1 & - & - \\
\hline $\begin{array}{ll}\text { contreforts } & (5) \\
\text { voute } & (6)\end{array}$ & $\begin{array}{r}58 \\
148\end{array}$ & $\begin{array}{l}45 \\
35\end{array}$ & $\begin{array}{l}10 \\
38\end{array}$ & $\frac{1}{26}$ & $\overline{5}$ & $\overline{2}$ & $\begin{array}{l}1 \\
6\end{array}$ & $\frac{1}{13}$ & $\overline{10}$ & $\overline{3}$ & $\overline{7}$ & $\bar{i}$ & - & - & $\overline{-}$ & $z$ & - & j & $\bar{i}$ & $=$ & - \\
\hline $\begin{array}{l}\text { voute } \\
\text { voute-multiple( }(7)\end{array}$ & $\begin{array}{r}148 \\
38\end{array}$ & 14 & 15 & 4 & 1 & - & 2 & 1 & 1 & - & - & - & - & - & - & - & $\overline{-}$ & 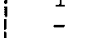 & - & $=$ & 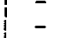 \\
\hline div.maçonnerié(8) & 12 & 6 & 3 & 2 & - & - & - & - & 1 & - & - & - & - & - & - & - & - & - & - & - & - \\
\hline maçonneries (4à8) & 632 & 249 & 152 & 58 & 9 & 4 & 68 & 36 & 29 & 7 & 7 & 3 & 3 & - & 1 & 1 & - & 3 & 2 & - & - \\
\hline mixtes divers (9) & 287 & 72 & 35 & 5 & - & - & 51 & 31 & 21 & 3 & - & 20 & 15 & 22 & 3 & - & 4 & 10 & 3 & 1 & 1 \\
\hline 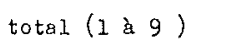 & 3714 & 775 & 354 & 97 & 14 & 4 & 1316 & 310 & 134 & 28 & 14 & 334 & 74 & 29 & 10 & 1 & 150 & 50 & 14 & 4 & 2 \\
\hline
\end{tabular}

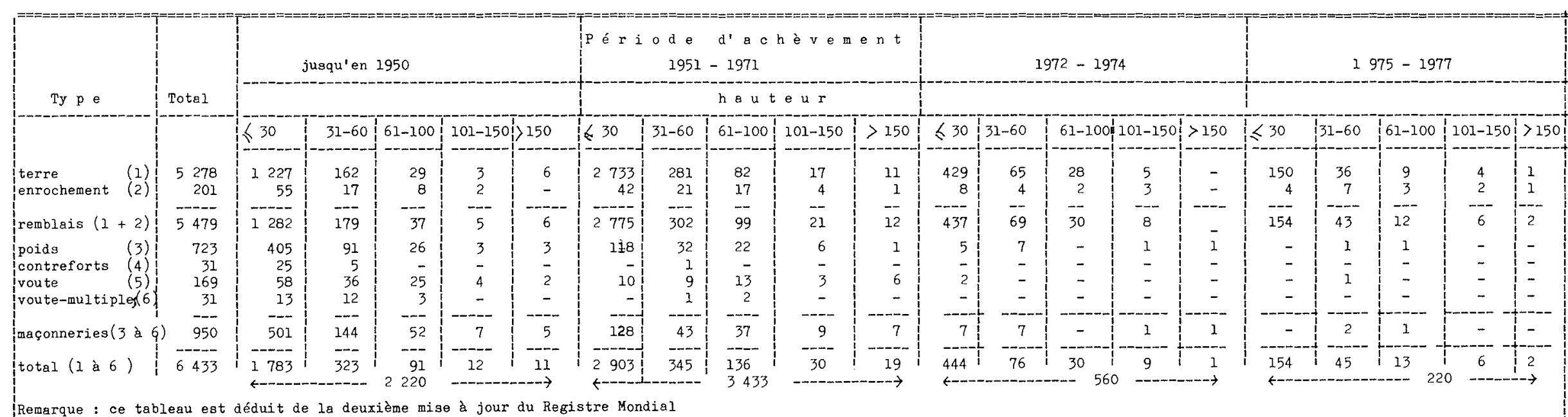


Ces tableaux sont assez cohérents sauf sur 2 points:

- en ce qui concerne les barrages de moins de $30 \mathrm{~m}$, pour les motifs déjà indiqués;

- en ce qui concerne les barrages de plus de $150 \mathrm{~m}$ antérieurs à 1950 : les erreurs manifestes signalées dans le renvoi de bas de page (18) expliquent au moins en partie cet écart.

\section{Autres pays}

Certains pays, dont le Japon, qui,avec 2003 ouvrages en service à la fin de 1977 , est le deuxième pays au monde (Chine exclue), ne font figurer exclusivement que des barrages de plus de $15 \mathrm{~m}$.

D'autres présentent un grand nombre d'ouvrages inférieurs à $15 \mathrm{~m}$.

Une analyse du Registre conduit aux résultats suivants :

\begin{tabular}{|c|c|c|c|c|c|c|c|c|c|c|}
\hline & $\begin{array}{l}\text { fin } \\
\text { période } \\
\text { consi- } \\
\text { dérée }\end{array}$ & $<15 \mathrm{~m}$ & $\begin{array}{c}\text { non } \\
\text { précisée }\end{array}$ & $\geqslant 15 \mathrm{~m}$ & total & $\begin{array}{c}\text { dont } \\
\text { anté- } \\
\text { rieurs } \\
\text { à } 1900\end{array}$ & $<15 \mathrm{~m}$ & $\begin{array}{c}\text { non } \\
\text { précisée }\end{array}$ & $\geqslant 15 \mathrm{~m}$ & total \\
\hline Afrique du Sud & 1977 & 29 & 3 & 285 & 317 & & - & - & 1 & 1 \\
\hline Albanie & 1971 & 2 & - & 23 & 25 & & - & - & - & - \\
\hline Algérie & 1977 & 1 & - & 20 & 21 & & - & - & 3 & 3 \\
\hline Allemagne & 1977 & 18 & - & 111 & 129 & & - & - & 7 & 7 \\
\hline Argentine & 1977 & 3 & - & 79 & 82 & & - & - & - & - \\
\hline Australie & 1977 & 28 & 2 & 288 & 318 & & 5 & 1 & 26 & 32 \\
\hline Autriche & 1977 & - & - & 53 & 53 & & - & - & - & - \\
\hline Belgique & 1977 & 1 & - & 10 & 11 & & - & - & - & - \\
\hline Brésil & 1977 & 48 & 3 & 332 & 383 & & - & - & - & - \\
\hline Bulgarie & 1969 & 1 & - & 65 & 66 & & - & - & - & - \\
\hline Canada & 1977 & 116 & 1 & 388 & 505 & & 2 & - & 1 & 3 \\
\hline Chili & 1977 & 4 & 1 & 54 & 59 & & 1 & - & 1 & 2 \\
\hline Chypre & 1977 & 2 & - & 25 & 27 & & - & - & - & - \\
\hline Colombie & 1977 & - & - & 28 & 28 & & - & - & - & - \\
\hline Corée & 1977 & 7 & 4 & 745 & 846 & & - & - & - & - \\
\hline Costa Rica & 1977 & - & - & 3 & 3 & & - & - & - & - \\
\hline Cote d'lvoire & 1977 & - & - & 3 & 3 & & - & - & - & - \\
\hline Danemark & 1977 & 3 & - & 3 & 6 & & - & - & - & - \\
\hline Egypte & 1974 & - & - & 3 & 3 & & - & - & & - \\
\hline Equateur & 1977 & - & - & 4 & 4 & & - & - & - & - \\
\hline Espagne & 1977 & 18 & 10 & 613 & 804 & & 29 & 1 & 20 & 50 \\
\hline Finlande & 1977 & 6 & - & 43 & 49 & & - & - & - & - \\
\hline France & 1977 & 13 & 18 & 353 & 384 & & - & 8 & 26 & 34 \\
\hline Ghana & 1971 & - & - & 3 & 3 & & - & - & - & - \\
\hline Gde Bretagne & 1977 & 78 & 1 & 444 & 523 & & 43 & 1 & 168 & 212 \\
\hline Grèce & 1977 & - & - & 9 & 9 & & - & - & - & - \\
\hline Hongrie & 1977 & 2 & - & 2 & 4 & & - & - & 1 & 1 \\
\hline Inde & 1977 & 80 & 17 & 658 & 755 & & 2 & 4 & 33 & 39 \\
\hline Indonésie & 1971 & - & - & 19 & 19 & & - & - & - & - \\
\hline Irak & 1977 & 1 & - & 4 & 5 & & - & - & - & - \\
\hline Iran & 1977 & - & - & 15 & 15 & & - & - & - & - \\
\hline Irlande & 1977 & - & 4 & 11 & 15 & & - & 3 & - & 3 \\
\hline Islande & 1977 & 2 & - & 7 & 9 & & - & - & - & - \\
\hline I talie & 1977 & 10 & - & 392 & 402 & & - & - & 7 & 7 \\
\hline Japon & 1977 & - & - & 2003 & 2003 & & - & - & 474 & 474 \\
\hline Jordanie & 1972 & - & - & 3 & 3 & & - & - & - & - \\
\hline Liban & 1974 & - & - & 5 & 5 & & - & - & - & - \\
\hline Luxembourg & 1977 & - & - & 3 & 3 & & - & - & - & - \\
\hline Malaisie & 1977 & - & 2 & 12 & 14 & & - & 2 & - & 2 \\
\hline Maroc & 1977 & - & - & 23 & 23 & & - & - & - & - \\
\hline Mexique & 1977 & 89 & - & 342 & 431 & & 9 & - & 6 & 15 \\
\hline Nigérla & 1974 & 1 & - & 4 & 5 & & - & - & - & - \\
\hline Norvège & 1977 & - & - & 212 & 212 & & - & - & 1 & 1 \\
\hline Nelle Zélande & 1977 & 2 & - & 64 & 66 & & - & - & 1 & 1 \\
\hline Pakistan & 1971 & 1 & - & 29 & 30 & & 1 & - & - & 1 \\
\hline Paraguay & 1974 & - & - & 1 & 1 & & - & - & - & - \\
\hline Pays Bas & 1977 & - & - & 7 & 7 & & - & - & - & - \\
\hline Pérou & 1971 & 21 & 6 & 27 & 54 & & 11 & 6 & 8 & 25 \\
\hline Philippines & 1977 & - & - & 8 & 8 & & - & - & - & - \\
\hline Pologne & 1977 & - & 1 & 19 & 20 & & - & - & - & - \\
\hline Portugal & 1977 & - & - & 52 & 52 & & - & - & - & - \\
\hline République Dominiquaine & 1977 & - & - & 2 & 2 & & - & - & - & - \\
\hline Rhodésie & 1974 & 3 & - & 64 & 67 & & - & - & - & - \\
\hline Roumanie & 1977 & 15 & - & 64 & 79 & & - & - & - & - \\
\hline Soudan & 1974 & - & - & 4 & 4 & & - & - & - & - \\
\hline Sri Lanka & 1977 & 29 & - & 35 & 64 & & 10 & - & 3 & 13 \\
\hline Suède & 1977 & - & - & 133 & 133 & & - & - & - & - \\
\hline Suisse & 1977 & - & - & 126 & 126 & & - & - & 2 & 2 \\
\hline Syrie & 1972 & - & - & 12 & 12 & & - & - & - & - \\
\hline
\end{tabular}




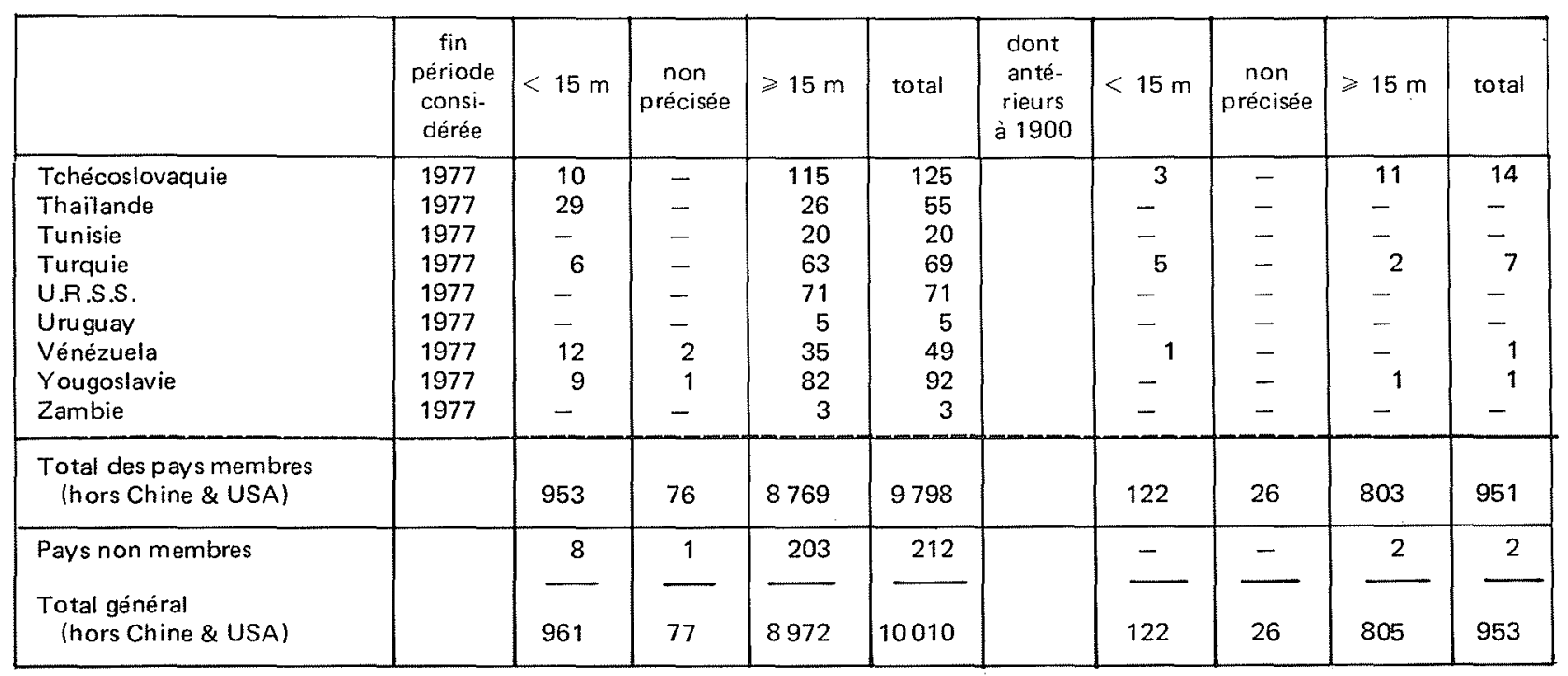

\section{ANNEXE 2 RUPTURES DE GRANDS BARRAGES ANTERIEURES AU 31/12/65}

Cette liste est pour l'essentiel déduite de l'ouvrage "Leçons tirées des accidents de barrages" publié par la Commission Internationale des Grands Barrages, compte tenu des corrections apportées par Niklaus Schnitter dans l'article "Sûreté statistique des barrages" paru dans la revue Wasser - Energie - Luft $n^{\circ} 5$ - 1976.

J'ai en outre introduit des renseignements tirés de l'exposé "Hazards and Risks to third parties from dams" présenté le 11/9/1978 par R.E. Coxon et F.N. Griffiths devant le Comité Britannique des Grands Barrages.

J'ai également pris en considération une communication espagnole présentée au Congrès de New Delhi (1979) de la C.I.G.B. ainsi qu'une communication indienne.

J'ai tenu également compte des recherches effectuées par le Service de la Production Hydraulique d'E.D.F. sur certains cas litigieux.
Ces trois dernières sources d'information m'ont conduit à apporter les modifications suivantes à la liste de la C.I.G.B. modifiée par Schnitter :

12 suppressions correspondant à :

- 5 accidents sans ruptures : Redbank Creek en Autralie, les 2 premiers accidents de Castlewood, le $2^{\mathrm{e}}$ accident de Sweetwater (U.S.A.) et le $2^{\mathrm{e}}$ accident de Bilberry (G.B.);

- 7 barrages inférieurs à $15 \mathrm{~m}$ : Goodrich (rompu 2 fois), Grass Valley, Hatchtown (2 premières ruptures), Lima Alamo, tous aux U.S.A. (le barrage d'Alamo, ajouté par Schnitter s'est vidé progressivement par ses fondations sans que le barrage ait été avarié).

7 adjonctions : Iruka au Japon, Bayless (1 ${ }^{\text {ère }}$ rupture en 1910), Cazadero et Gallinas aux U.S.A. (l'enquête de la C.I.G.B. comportait effectivement l'erreur relevée par Schnitter, mais un barrage en maçonnerie et bois construit en 1910 a vu sa partie en bois emportée par une crue en 1957), Oros au Brésil (barrage en cours de construction submergé par une crue), Hyokiri en Corée (bien que ce barrage ait peut-être une hauteur inférieure à $15 \mathrm{~m}$; l'enquête de la C.I.G.B. ne portait pas sur ce pays), Torrejon Tajo en Espagne (rupture d'une vanne en cours de construction : 30 morts). 


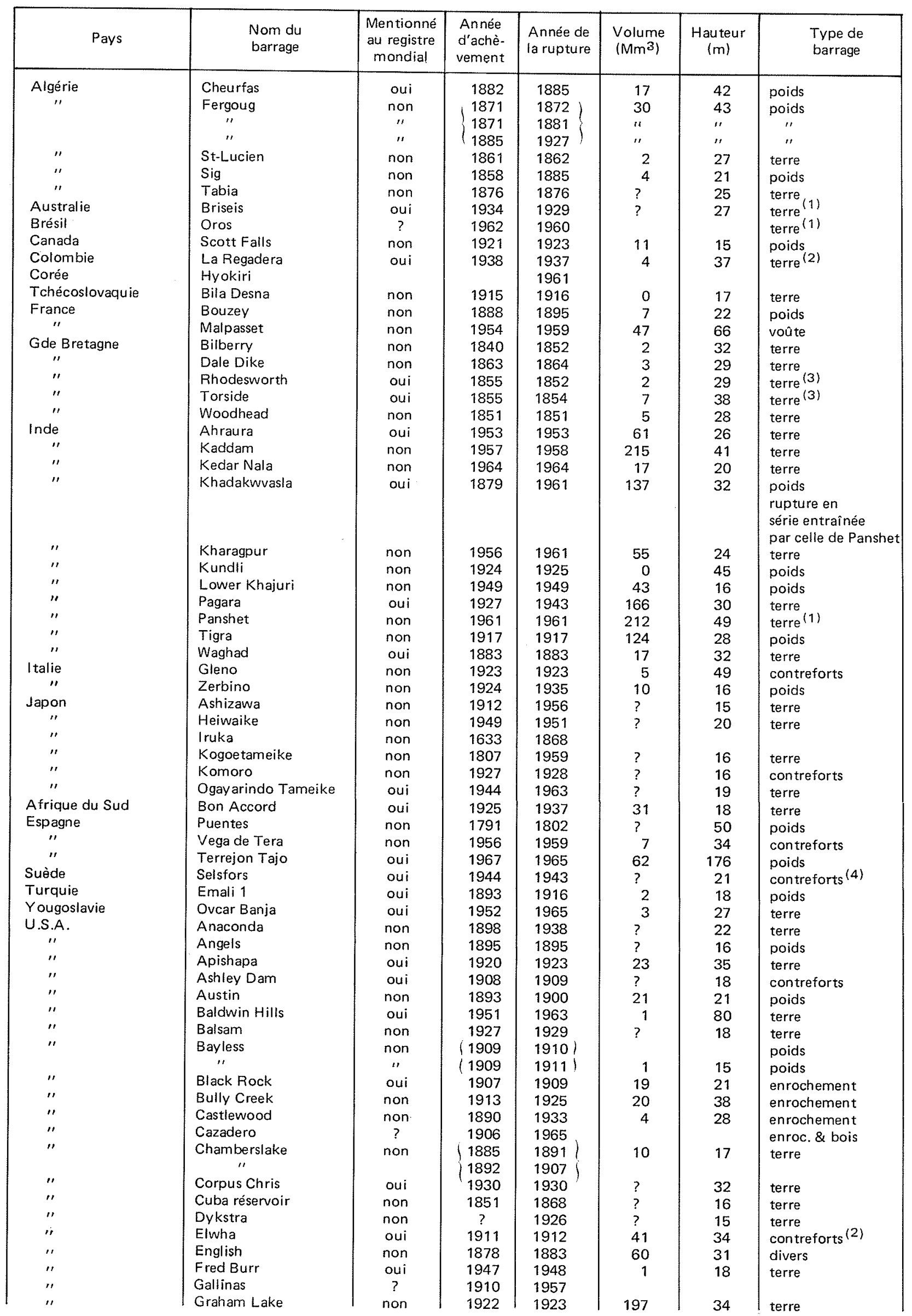




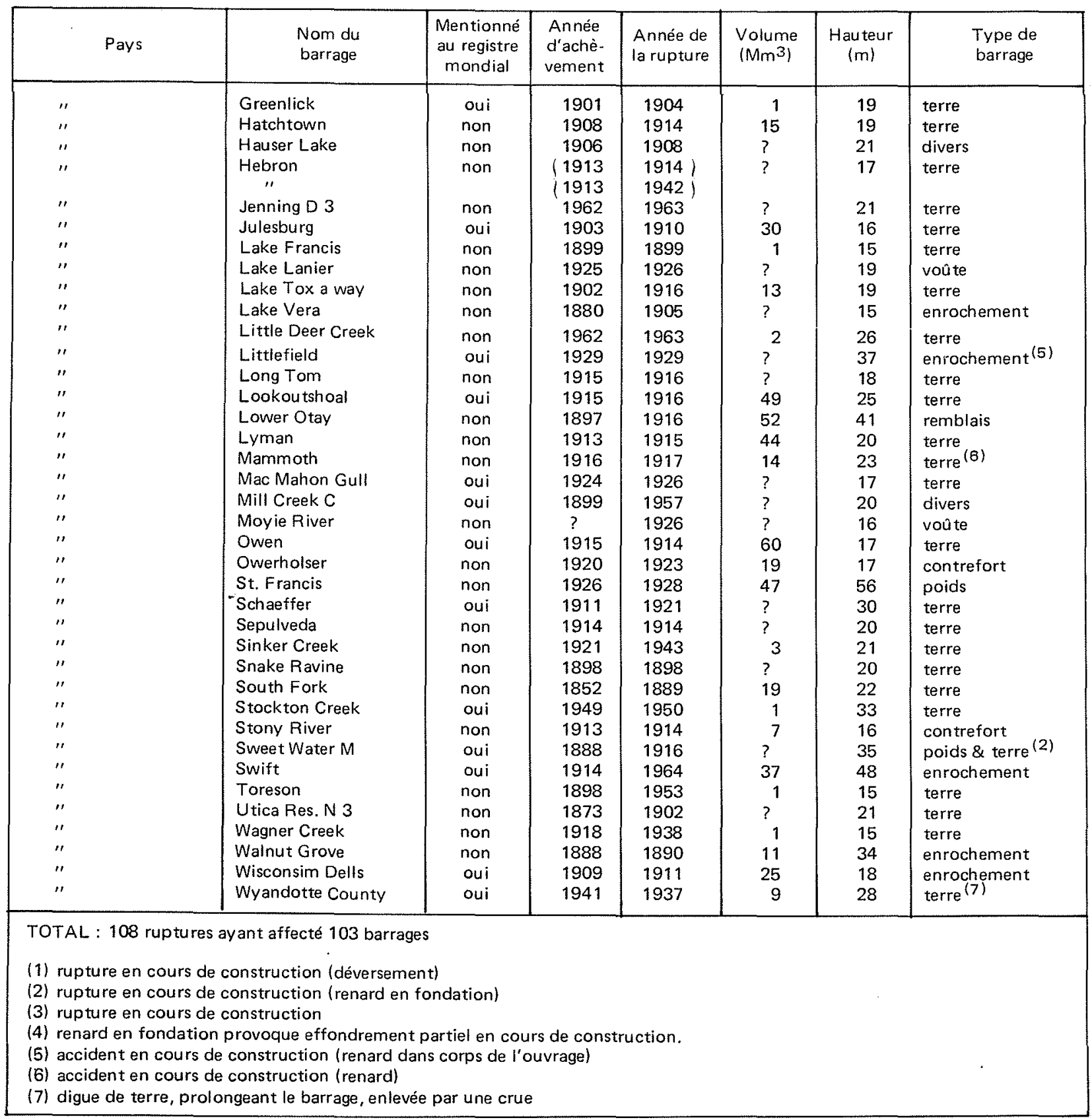


ANNEXE 3

RUPTURES DE GRANDS BARRAGES POSTERIEURES AU $1^{\text {er }}$ JANVIER 1966

\begin{tabular}{|c|c|c|c|c|c|c|}
\hline Nom du barrage & Pays & $\begin{array}{c}\text { Date } \\
\text { d'achèvement }\end{array}$ & Hauteur & Type & $\begin{array}{l}\text { Date de } \\
\text { rupture }\end{array}$ & $\begin{array}{l}\text { Cause de la } \\
\text { rupture }\end{array}$ \\
\hline Pardo & Argentine & 1940 & $15 m$ & Enroch. & $12 / 69$ & $\begin{array}{l}\text { Crue } \\
\text { Défaut d'entretien }\end{array}$ \\
\hline Armando Salles de Oliveira & Brésil & 1958 & 41 ou $32 \mathrm{~m}$ & Terre & $20 / 01 / 77$ & Submersion \\
\hline Euclides de Cunha & Brésil & 1960 & $63 \mathrm{~m}$ & Terre & $20 / 01 / 77$ & Submersion \\
\hline Del Monte & Colombie & $\begin{array}{l}\text { Non } \\
\text { répertorié }\end{array}$ & & & 10/76 & $\begin{array}{l}\text { Rupture d'une } \\
\text { vanne ( } 80 \text { morts) }\end{array}$ \\
\hline Odiel & Espagne & $1970(?)$ & $35 \mathrm{~m}$ & Enroch. & 1970 & (1) \\
\hline Nanaksagar & Inde & 1962 & $15,6 \mathrm{~m}$ & Terre & $07 / 09 / 67$ & Renard (100 morts) \\
\hline Chitauni & Inde & $\begin{array}{l}\text { Non } \\
\text { répertorié }\end{array}$ & & Enroch. & 68 & \\
\hline Dhanibara & Inde & 1975 & $20,7^{(2)} \mathrm{m}$ & Terre & $04 / 06 / 76$ & Submersion \\
\hline Manivali & Inde & 1976 & $18,4^{(2)} \mathrm{m}$ & Terre & $19 / 07 / 76$ & $1^{\mathrm{er}}$ remplissage \\
\hline Macchu 2 & Inde & 1973 & $26 \mathrm{~m}$ & Terre & $08 / 79$ & Submersion \\
\hline Sempor & Indonésie & $\begin{array}{l}\text { commencé } \\
\text { en } 1967\end{array}$ & $53,65 \mathrm{~m}$ & Enroch. & $12 / 67$ & $\begin{array}{l}\text { Rupture en cours } \\
\text { de chantier } \\
\text { ( } 200 \text { morts) }\end{array}$ \\
\hline Wadigatara & Lybie & $1975(?)$ & $39 m$ & Terre & 1975 & $\begin{array}{l}\text { Renard } \\
\text { 1er remplissage }^{\text {er }}\end{array}$ \\
\hline La Laguna & Mexique & 1912 & $17 \mathrm{~m}$ & Terre & 1969 & Renard \\
\hline Bolan & Pakistan & 1961 & $19 \mathrm{~m}$ & Terre & $09 / 76$ & Submersion \\
\hline San to Thomas & Philippines & $\begin{array}{l}\text { commencé } \\
\text { en } 1975\end{array}$ & & Terre & 1976 & $\begin{array}{l}\text { Crue (typhon sur le } \\
\text { chantier ( } 80 \text { morts) }\end{array}$ \\
\hline Emery & U.S.A. & 1850 & $15,30 \mathrm{~m}$ & Terre & 1966 & $\begin{array}{l}\text { Renard } \\
\text { (corrosion conduite) }\end{array}$ \\
\hline $\begin{array}{l}\text { Whitewater Brook } \\
\text { (Upper) }\end{array}$ & U.S.A. & 1943 & $18,6 \mathrm{~m}$ & Terre & $04 / 05 / 72$ & $\begin{array}{l}\text { Affouillements le } \\
\text { long du chenal de } \\
\text { déversement }\end{array}$ \\
\hline Sheep Creek & U.S.A. & 1969 & $18 \mathrm{~m}$ & Terre & $08 / 05 / 70$ & $\begin{array}{l}\text { Renard le long de la } \\
\text { conduite d'évacua- } \\
\text { tion des crues }\end{array}$ \\
\hline Lake Barcroft & U.S.A. & 1913 & $21 \mathrm{~m}$ & $\begin{array}{l}\text { Poids et } \\
\text { terre }\end{array}$ & $21 / 06 / 72$ & $\begin{array}{l}\text { Brèche dans partie } \\
\text { terre (crue) }\end{array}$ \\
\hline Walter Bouldin & U.S.A. & 1967 & $50 \mathrm{~m}$ & Terre & $10 / 02 / 75$ & $\begin{array}{l}\text { ? - Attribué à un } \\
\text { glissement progres- } \\
\text { sif du talus amont }\end{array}$ \\
\hline Teton & U.S.A. & 1976 & $120 \mathrm{~m}$ & Terre & $05 / 06 / 76$ & $\begin{array}{l}\text { Renard } \\
\text { (11 morts) }\end{array}$ \\
\hline \multicolumn{7}{|c|}{ TOTAL : 21 ruptures ayant affecté 21 barrages. } \\
\hline $\begin{array}{l}\text { dure en cours de cc } \\
\text { dessus du thalweg. }\end{array}$ & & du ba & & & & \\
\hline
\end{tabular}

